Meta

Journal des traducteurs

Translators' Journal

\title{
Dificultades, estrategias y recursos en la traducción de estados financieros: fuentes normativas y textos paralelos
}

\section{Marta García González}

Volume 64, numéro 2, août 2019

URI : https://id.erudit.org/iderudit/1068204ar

DOI : https://doi.org/10.7202/1068204ar

Aller au sommaire du numéro

Éditeur(s)

Les Presses de l’Université de Montréal

ISSN

0026-0452 (imprimé)

1492-1421 (numérique)

Découvrir la revue

Citer cet article

García González, M. (2019). Dificultades, estrategias y recursos en la traducción de estados financieros: fuentes normativas y textos paralelos. Meta, 64(2), 491-513. https://doi.org/10.7202/1068204ar
Résumé de l'article

Sur la base du dépouillement bibliographique dans le domaine de la traduction comptable, l'article analyse les besoins de traduction générés dans ce domaine de l'économie, ainsi que les principales difficultés liées au transfert de ce type de textes et des stratégies de traduction les plus appropriées pour les affronter. L'impossibilité d'utiliser des équivalents fonctionnels dans de nombreux cas est envisagée, en raison de la coexistence de normes comptables différentes et de la difficulté de déterminer les besoins et les attentes des utilisateurs des traductions. Ensuite, l'utilité des sources normatives, ainsi que des textes et des corpus parallèles en tant que ressources pour l'extraction terminologique et la clarification des ambiguïtés dans la traduction des états financiers, est évaluée. À cet effet, deux types de sources sont analysés, les Normes Internationales d'Information Financière (IFRS) dans leurs versions originales et adaptées à l'UE, et des états financiers des entreprises espagnoles, britanniques et américaines. Les résultats soulignent la nécessité de promouvoir l'acquisition de compétences thématiques en la matière comme une étape essentielle pour une gestion satisfaisante du processus documentaire et de traduction. 


\title{
Dificultades, estrategias y recursos en la traducción de estados financieros: fuentes normativas y textos paralelos
}

\author{
MARTA GARCÍA GONZÁLEZ \\ Universidade de Vigo, Vigo, España \\ mgarciag@uvigo.es
}

\section{RÉSUMÉ}

Sur la base du dépouillement bibliographique dans le domaine de la traduction comptable, l'article analyse les besoins de traduction générés dans ce domaine de l'économie, ainsi que les principales difficultés liées au transfert de ce type de textes et des stratégies de traduction les plus appropriées pour les affronter. L'impossibilité d'utiliser des équivalents fonctionnels dans de nombreux cas est envisagée, en raison de la coexistence de normes comptables différentes et de la difficulté de déterminer les besoins et les attentes des utilisateurs des traductions. Ensuite, l'utilité des sources normatives, ainsi que des textes et des corpus parallèles en tant que ressources pour l'extraction terminologique et la clarification des ambiguïtés dans la traduction des états financiers, est évaluée. À cet effet, deux types de sources sont analysés, les Normes Internationales d'Information Financière (IFRS) dans leurs versions originales et adaptées à l'UE, et des états financiers des entreprises espagnoles, britanniques et américaines. Les résultats soulignent la nécessité de promouvoir l'acquisition de compétences thématiques en la matière comme une étape essentielle pour une gestion satisfaisante du processus documentaire et de traduction.

\begin{abstract}
Based on a literature review in the field of accounting translation, the article discusses the actual needs for translation of financial statements, as well as the main difficulties associated with the transfer of this type of text. Reference is made to the impossibility of using functional equivalents in many cases, due to the coexistence of different accounting standards across countries and to the difficulty of determining the needs and expectations of translations' end users. Next, the article evaluates the usefulness of legal sources, and of parallel texts and corpora as resources for terminology extraction and ambiguity clarification in the translation of financial statements. To this end, two types of sources are analyzed, namely International Financial Reporting Standards (IFRS) and financial statements of Spanish, British and US companies. The results confirm the need to encourage the acquisition of subject competences by trainees as an essential supplement to the info-mining and translation competences.
\end{abstract}

\section{RESUMEN}

A partir de la revisión bibliográfica en el ámbito de la traducción contable, el artículo analiza las necesidades de traducción que se generan en este campo de la economía, así como las principales dificultades asociadas a la transferencia de este tipo de textos y las estrategias de traducción más adecuadas para hacerles frente. Se valora la imposibilidad de utilizar equivalentes funcionales en muchos casos, debido a la coexistencia de normativas contables diferentes y a la dificultad que supone determinar las necesidades y expectativas de los usuarios de las traducciones. A continuación, se evalúa la utilidad de las fuentes normativas, así como de los textos y corpus paralelos como recursos para la extracción terminológica y la aclaración de ambigüedades en la traducción de estados 
financieros. Para ello, se analizan principalmente dos tipos de fuentes, las Normas Internacionales de Información Financiera (NIIF) en sus versiones original y adaptada a la UE, y una selección de estados financieros de empresas españolas, británicas y estadounidenses. Los resultados apuntan hacia la necesidad de promover la adquisición de competencias temáticas en la materia como paso imprescindible para gestionar de manera satisfactoria el proceso documental y de traducción.

\section{MOTS CLÉS/KEYWORDS/PALABRAS CLAVE}

comptabilité, états financiers, sources normatives, textes parallèles, traduction accounting, financial statement, legal sources, parallel texts, translation contabilidad, estados financieros, fuentes normativas, textos paralelos, traducción

Para que los estados financieros que atraviesan las fronteras nacionales sean realmente útiles, no es suficiente con que se presenten en diferentes idiomas

y monedas, sino que, más bien, deberían ser traducidos de forma que se transmitieran todas las circunstancias, hechos subyacentes y consecuencias a los usuarios, cuyas experiencias y formación en materia de información económico-financiera difieren de las de la entidad informadora.

(Laínez 1993: 80)

\section{Introducción}

La traducción de documentos contables constituye uno de los ámbitos de especialización dentro de la traducción económica que mayores retos plantea para el traductor, debido a la complejidad de la propia actividad contable, pero también a la existencia de importantes diferencias entre sistemas de contabilidad y a la multiplicidad de normas que se pueden encontrar dentro de un mismo sistema. A modo de ejemplo, puede considerarse el caso de España, donde desde 2005 (Reglamento UE $1606 / 2002$ ) las empresas cotizadas deben formular las versiones consolidadas de sus estados financieros según las Normas Internacionales de Información Financiera $(\mathrm{NIIF})^{1}$ en versión europea (NIIF-UE) ${ }^{2}$. Las demás empresas pueden elaborar sus estados consolidados siguiendo estas normas o con arreglo al Plan General Contable (PGC). Por el contrario, las cuentas independientes de cada empresa deben elaborarse según el PGC aplicable, ya sea el estándar (Real Decreto 1514/2007) o el específico para pymes (Real Decreto 1515/2007). Por su parte, las empresas que operan en determinados sectores han de ajustarse a normas específicas, como las entidades de crédito, que se rigen por la Circular 4/2004, de 22 de diciembre, del Banco de España, modificada por la Circular 6/2008.

Cabe preguntarse cómo será capaz un traductor de manejarse entre este entramado de normas, cuando además debe realizar el mismo ejercicio por duplicado, para determinar la normativa contable más actualizada del sistema asociado a cada una del par de lenguas implicado en la traducción. Esto en el caso ideal de que el encargo de traducción determine cuál es el país destinatario de su traducción, dado que las variables idioma y país no suelen ir parejas. Con frecuencia puede incluso darse el caso de que la traducción no tenga, en realidad, un destinatario único, sino que un informe anual se traduzca al inglés para su difusión entre inversores procedentes de diferentes países con sistemas contables también diferentes.

El presente trabajo tiene como objetivo reflexionar sobre la traducción de estados financieros en torno a tres cuestiones centrales. En primer lugar, dado que los estados 
financieros son documentos generados por una empresa y dirigidos principalmente a la administración pública y a los administradores y socios o accionistas de la empresa, intentaremos determinar si existe una necesidad real de traducción y, en caso de que así sea, cuáles son sus principales destinatarios y la función que ha de satisfacer. En segundo lugar, nos centraremos en sus principales dificultades y en las estrategias de traducción más productivas, atendiendo al grado de equivalencia que sea posible obtener entre los conceptos recogidos en el TO y en el TM. Por último, procederemos a evaluar los materiales y recursos que pueden ayudar al traductor en su trabajo, que estarán en función de la estrategia adoptada pero también de la competencia temática del propio traductor.

Para dar respuesta a las cuestiones planteadas recurriremos en primer lugar a la revisión bibliográfica, en la que se analizarán una selección trabajos dedicados a la traducción de documentos contables. A continuación, se realizará una revisión de los recursos más relevantes de los que dispone el traductor, con especial atención a la legislación contable y a los textos paralelos, que nos permita valorar las soluciones que cada uno puede aportar a la traducción final del producto.

\section{Estado de la cuestión}

Aunque los estudios dedicados a la traducción de documentos contables son escasos, su número ha aumentado en los últimos años, en parte propiciados por los movimientos de armonización contable promovidos desde la Fundación IFRS. Por su ámbito de procedencia, los trabajos se dividen en dos grandes bloques, los elaborados desde el ámbito de los estudios de traducción y los elaborados desde el ámbito de la contabilidad. Esta doble procedencia nos permite abordar el estudio de la traducción contable desde dos perspectivas claramente diferenciadas, la de los emisores de la traducción y la de los potenciales emisores del original y potenciales receptores de la traducción.

\subsection{Por qué traducir estados financieros}

Jeanjean, Lesage et al. (2010), en un estudio sobre informes anuales de los ejercicios 2003 y 2004 de 27 países diferentes de habla no inglesa, determinaron que en torno a un $50 \%$ de las empresas publicaban sus cuentas tanto en la lengua local como en inglés. Entre los factores que determinaban el empleo del inglés se encontraban el tamaño de la empresa, la dispersión geográfica de sus accionistas, el nivel de internacionalización de sus ventas, su presencia en bolsas de países de habla inglesa y las necesidades de financiación externa. Una búsqueda de los estados financieros de 2015 de las empresas españolas que componían entonces el IBEX35 indica que a excepción de ArcelorMittal, que publica sus informes directamente en inglés, todas ellas emitieron una versión inglesa como complemento a la versión española oficial. Campbell, Beck et al. (2005) presentaban iguales resultados al analizar los informes de las 35 empresas con mayor cotización en la Bolsa de Frankfurt.

Carmona (2012) revisa las necesidades de traducción de estados financieros entre Francia y España en procesos vinculados a la internacionalización de empresas y llega a la conclusión de que la traducción es necesaria en Francia para la constitución de sucursales, mientras que puede ser recomendable, tanto en Francia como en España, para las actividades relacionadas con fusiones, participaciones en sociedades, alian- 
zas y cooperaciones. Exigencias similares relativas a la traducción de cuentas anuales se constatan en otros estados, como Bélgica, Reino Unido, Estados Unidos o Emiratos Árabes Unidos. Por otro lado, Carmona (2012: 161) afirma que en España no es necesario que las empresas extranjeras con sucursales en el país traduzcan sus estados financieros al español, ya que, aunque existe la obligación de que la sociedad matriz deposite los estados en el registro mercantil al que pertenece la sucursal, la legislación no establece que estos deban estar redactados en un idioma concreto. Sin embargo, existe jurisprudencia que determina la obligatoriedad de presentar en el registro una traducción al español de las cuentas, concretamente una resolución de enero de 2011, de la Dirección General de los Registros y del Notariado, que con base en el artículo 37 del Reglamento Hipotecario y en el artículo 4 de la Undécima Directiva 89/666/CEE del Consejo, de 21 de diciembre de 1989, establece que:

la presunción de conocimiento general y su oponibilidad frente a terceros exigen que el contenido de los asientos puedan ser conocidos por todos, lo que no podría ocurrir de estar redactados en lengua diferente a la que es oficial en el territorio donde radica el Registro. (Ministerio de Justicia 2011)

La traducción de estados financieros parece por tanto una práctica habitual en el caso de empresas cotizadas y una necesidad legal en el caso de empresas internacionalizadas que quieren constituir sucursales en otros países. No suele tratarse de una práctica común entre las pymes, que bien suelen limitar su actividad al ámbito estatal, bien centran sus acciones de internacionalización en actividades de exportación de manera directa o a través de representantes independientes (Johanson y Vahlne 1977). Por otra parte, en los paraísos fiscales, así como en países pequeños, suele estar permitido que las empresas utilicen el inglés para sus informes. Así sucede en los Países Bajos, donde las empresas pueden presentar sus estados financieros en inglés, francés, alemán o flamenco; o en Luxemburgo, donde pueden formularse en inglés, además de en francés y en alemán (Clark 1994).

Cabe hacer referencia, además, a la relevancia de la traducción desde el punto de vista de la comprensión. Pese al papel del inglés como lengua franca en la economía, es importante pensar que el grado de comprensión de un TO en inglés para un hablante no nativo puede ser inferior al de una posible traducción a su lengua. Doupnik y Richter (2003: 30) sugieren que los hablantes no nativos de inglés pueden sentir menos seguridad al interpretar términos que expresen incertidumbre, muy habituales en contabilidad, ante la versión inglesa de las NIIF que ante una versión traducida. Holthoff, Hoos et al. (2015) demostraron que el uso de la traducción al alemán de la NIC 24 (Información a revelar sobre partes vinculadas) mejoraba la toma de decisiones de los usuarios frente al uso del original inglés.

\subsection{Cómo traducir estados financieros}

La revisión bibliográfica revela que existe cierta preocupación por la calidad de las traducciones. Así, Courtis y Hassan (2002) afirman que, en algunos casos, la traducción al inglés puede resultar más difícil de leer que el original en lengua vernácula, lo que puede crear un coste indirecto. Por su parte, Jeanjean, Lesage et al. (2010: 202) afirman que, si bien publicar una traducción al inglés de un informe anual puede aumentar la base de inversores, también puede implicar el riesgo de enviar un mensaje menos articulado a los hablantes de habla inglesa. 
En general, desde el ámbito de la economía, los estudios sobre la traducción contable se han centrado en el estudio y valoración de las traducciones de las Directivas UE y de las NIIF, generadas por su adopción como normativa aplicable en la contabilidad de las empresas cotizadas de los Estados Miembros. Así, Hellman, Perera et al. $(2010)^{3}$ analizan la versión original inglesa y la traducción alemana de las NIIF y determinan que su contenido no siempre es equivalente. También Nobes (2015: 416) menciona errores en la traducción de la Cuarta Directiva 78/660/CEE del Consejo, la primera directiva dirigida a la armonización contable de las sociedades de capital de la Unión. En este caso, el error se detecta en la versión inglesa realizada desde el original francés, y en su posterior trasposición a la legislación británica. Concretamente, el término francés passif del balance se tradujo al inglés como liability, debido a la inexistencia de un término único equivalente en lengua inglesa para el término francés, y sin tener en cuenta que passif incluía no solo obligaciones sino también el capital social de la empresa.

Entre los trabajos más detallados elaborados desde el ámbito de la contabilidad destacan Baskerville y Evans (2011a, 2011b) y Kettunen (2017), basados en entrevistas, a redactores y traductores de manuales de contabilidad en el primer caso y a agentes implicados en el proceso de traducción de las normas en el segundo y tercero. Con todo, son aún escasos los trabajos que analicen traducciones de informes financieros reales y problemas concretos de traducción de conceptos o términos especializados. Archer y McLeay (1991) comparan cuentas de empresas europeas en 11 lenguas diferentes y Mourier (2004) revisa los principales retos que plantea la traducción de informes del danés al inglés y repasa cuestiones como la variedad de inglés que se debe seleccionar en función del receptor del TM.

Desde el ámbito de los estudios de traducción, Pizarro Sánchez (2000) dedica su tesis doctoral al estudio del género textual 'memoria anual' y de sus traducciones al inglés, mientras Carmona (2012) centra la suya en el análisis textual y la traducción de balances del español al francés. En el marco de la formación de traductores, Alcalde (2013) realiza una propuesta didáctica de la traducción de un balance, combinada con un corpus bilingüe ad hoc de textos paralelos, mientras que García y Veiga (2014) proponen una experiencia de indagación guiada y traducción colaborativa de cuentas anuales basada en la explotación de fuentes normativas y textos paralelos.

Recientemente, Nobes y Stadler $(2017)^{4}$ realizan un estudio sobre la traducción del concepto impairment en varias versiones de la NIC 36 (Deterioro del valor de los activos) y sobre la traducción al inglés del mismo concepto en un corpus de más de 300 cuentas anuales de empresas cotizadas. Los autores llegan a la conclusión de que el desarrollo de la terminología contable puede provocar problemas incluso en la redacción original de las normas y que una traducción que no sea exacta puede llevar a interpretaciones incorrectas de la norma y a su aplicación de diferente forma en cada país, lo que dificulta la armonización. El uso de terminología no equivalente en las cuentas anuales de las empresas de estos países hace que al traducirlas al inglés se empleen términos diferentes al empleado originalmente en la normativa aplicable. Por este motivo, para la traducción de normas contables del inglés a otras lenguas defienden la equivalencia formal, siempre que sea posible, si bien no proponen soluciones en aquellos casos en los que no lo sea. Por el contrario, para la traducción de cuentas anuales de otras lenguas al inglés, proponen una estrategia basada en la interpretación del original para inferir cuál era el término utilizado inicialmente en la norma aplicada. 
Sus conclusiones no parecen estar en consonancia con las alcanzadas por Baskerville y Evans (2011a), quienes en su estudio sobre la traducción a las lenguas de la UE de las NIIF llegan a las siguientes conclusiones:

- No es posible alcanzar una equivalencia exacta a través de la traducción. Cuando un concepto no forma parte de una cultura, su traducción no es significativa, independientemente del modo en que se traduzca.

- El alcance semántico de los términos no tiene por qué ser necesariamente el mismo de una lengua a otra, ni siquiera dentro de lo que denominamos terminología especializada.

- Existen problemas que están asociados a diferencias en la estructura de las lenguas.

- Tanto los problemas como las soluciones difieren de unas familias lingüísticas a otras.

De lo anterior podemos inferir que no todos los problemas que surgen en la traducción de informes financieros son de carácter terminológico, como destaca asimismo Kettunen (2017: 52) quien destaca también los problemas vinculados a la interpretación del texto original y a la presencia de ambigüedades que no siempre se pueden mantener en la traducción.

\subsubsection{Terminología especializada}

Algunos autores, como Huerta, Petrides et al. (2013: 4), afirman que en contabilidad existe una terminología técnica que refleja un significado conceptual, por lo que su traducción es más fácil que la del lenguaje general. Esta puede ser la causa por la cual, al estudiar las traducciones de las NIIF a los diferentes idiomas de la Unión Europea, muchos autores (Doupik y Richter 2003; Evans 2004; Tsakumis, Campbell et al. $2009^{5}$ ) han centrado su atención principalmente en conceptos subjetivos relacionados con la lengua general, como las nociones true and fair value, material, likely, considerable o remote. Algunos de ellos son términos que adquieren un significado específico cuando pasan de la lengua general a la de especialidad, como es el caso de material. Sin embargo, la principal dificultad en todos ellos reside en que establecen una gradación, por lo que requieren que el lector tome una decisión sobre el alcance de su significado.

Con todo, como afirman Baskerville y Evans (2011a), en las ciencias sociales, la definición de terminología específica no se deriva de manera sistemática ni carece de ambigüedad, sino que por el contrario puede provocar mayor controversia que en las ciencias naturales. No debemos olvidar que tratamos con sistemas contables diferentes, por lo que los conceptos (cuentas, partidas) utilizados en ellos no tienen por qué ser siempre equivalentes. Debemos referirnos aquí a la dicotomía entre equivalente funcional a nivel conceptual desde el punto de vista del derecho comparado y equivalencia funcional a nivel textual desde la perspectiva traductológica (García 2017). Resultan evidentes las dificultades que entraña encontrar un equivalente funcional entre sistemas basados en tradiciones contables diferentes. Dahlgren y Nilsson (2012: 52) proporcionan un ejemplo claro extraído del sistema contable sueco, que carece del concepto income, entendido como la suma de revenues y gains. De igual forma, Alexander y Nobes (2001: 210) analizan las diferencias entre los conceptos Abschreibung en alemán, amortissement y dépréciation en francés, y amortisation, depreciation e impairment en inglés. De acuerdo con los autores, la lengua inglesa dispone de tres términos diferenciados para referirse a la amortización de activos 
tangibles (depreciation), a la amortización de activos intangibles (amortization) y al deterioro o pérdida de valor de los activos (impairment) En francés, sin embargo, sólo se emplea el término amortissement para hacer referencia a la amortización contable de activos, sean tangibles o intangibles, mientras que dépréciation se emplea para denominar las pérdidas de valor, sean o no por deterioro. Por último, en alemán, el término Abschreibung puede aludir tanto al deterioro como a la amortización contable. Sin embargo, tampoco la existencia de un equivalente funcional en el sistema de llegada implica que utilizarlo garantice la equivalencia funcional entre los textos origen y meta. Hellman, Perera et al. (2010) ejemplifican este problema con respecto a la traducción al alemán de las NIIF, en concreto a la elección de un término alemán para traducir el término inglés asset. Tradicionalmente, la contabilidad alemana se refería al concepto activo con el término Vermögensgegenstand, que podría haber sido elegido como equivalente funcional para traducir asset en el contexto de las NIIF. No obstante, un análisis detallado del alcance del término alemán, en atención a los principios contables del sistema germano (continental), frente al análisis del término asset propio de la contabilidad anglosajona, indicó que este último tenía un mayor alcance semántico (incluía el fondo de comercio y las partidas diferidas, no incluidos en el concepto alemán). Esto, junto con otras consideraciones relativas a la interpretación de ambos términos, hizo que los traductores optasen por acuñar un nuevo término, Vermögenswert, no empleado hasta entonces en el código de comercio alemán. En estos casos, Evans considera que:

[i]f a concept or an idea is translated into another language, and the signifier used is already linked - in the minds of the native speakers of that language - with another concept or idea, it is likely that they will confuse the translation with the idea with which they are already familiar. (Evans 2004: 226)

En ese mismo sentido apuntaban algunas de las opiniones de traductores profesionales recogidas por Baskerville y Evans, según las cuales,

non-translation of terminology ('maintain the original words') with explanation of the original accounting context may be easier than to translate concepts with (not) equivalent terms of a second language. (Baskerville y Evans 2011a: 32-33)

Puede suceder, por último, que se introduzcan en las NIIF términos que designan conceptos nuevos, dirigidos a dar respuesta a problemas que nunca han surgido en muchos de los países receptores. Estos casos, en los que la utilización de un equivalente se vuelve totalmente imposible, suscitan aún mayor problema, pues como afirma Zeff (2007: 296) incluso si se traducen «as accurately as practical [...] the words may be understood, but the concept may not be understood».

Cuando el uso de un equivalente funcional no garantiza la equivalencia funcional, o cuando no existe siquiera un concepto semejante en la cultura meta, se hace necesario recurrir a otras estrategias que garanticen la transferencia del contenido. En su análisis de estados financieros traducidos, Archer y McLeay (1991: 357-360) identifican como estrategias varios niveles de omisión, introducción de neologismos, ampliaciones, reformulaciones y aproximaciones y la conservación del término original. Baskerville y Evans (2011b: 28) identifican las mismas estrategias en la traducción de las NIIF, con excepción de las omisiones y la creación de neologismos. Respecto a la conservación del término original, habitual en la traducción de otro tipo de textos incluso en el ámbito de la economía, es criticada por algunos autores, 
como Smith (1995: 33-34), por entender que contribuye a crear un lenguaje elitista además de dificultar la comunicación.

\subsubsection{Problemas derivados de las diferencias estructurales entre las lenguas}

Como hemos visto, Baskerville y Evans (2011a) concluyen que no todos los problemas de la traducción contable son terminológicos y que existen dificultades asociadas a diferencias en la estructura de las lenguas. En ocasiones, estas dificultades se revelan incluso más difíciles de resolver que las terminológicas, ya que exigen un mayor conocimiento tanto de la lengua como del tema tratado (Kettunen 2017: 48).

No todas las lenguas presentan la misma complejidad sintáctica. Por ejemplo, la sintaxis de la lengua española tiende a ser más compleja que la inglesa, lo que puede requerir un esfuerzo de simplificación sintáctica sin simplificación del contenido en la traducción inversa. Sin embargo, el discurso especializado inglés puede presentar también problemas asociados a la complejidad de las oraciones y a la falta de puntuación. En muchos casos, resulta útil dividir la oración en sintagmas y analizarlos sintácticamente para establecer las relaciones explícitas e implícitas entre ellos antes de reconstruir el contenido en la lengua de llegada.

Cuando los sintagmas del original se relacionan de forma que puedan contener ambigüedades que el traductor no es capaz de despejar, como establecer cuál es el antecedente exacto de un pronombre de relativo, o a qué sustantivos califica un adjetivo antepuesto, habrá que determinar si el contexto permite deshacer la ambigüedad o si puede ser recomendable recurrir a los recursos de la LM para mantenerla. En este sentido, es importante la afirmación de Evans, Baskerville et al. (2015), quienes recuerdan que las NIIF son normas basadas en principios, que se caracterizan por utilizar la ambigüedad para permitir cierto margen a la interpretación y aplicación en distintos entornos empresariales. Por otra parte, deshacer ambigüedades requiere bien un profundo dominio de la contabilidad, bien un intenso esfuerzo de documentación. El uso de diccionarios difícilmente puede ayudar al traductor en este caso, por lo que deberá manejar recursos alternativos como fuentes normativas o fuentes temáticas, que le requerirán una mayor inversión de tiempo.

\subsubsection{Problemas de variación lingüística en el TO y en la LM}

Al igual que sucede en la lengua general, el lenguaje especializado presenta diferencias relevantes entre las variedades británica y estadounidense. En la terminología contable, los pares financial year/fiscal year, turnover/sales, stock/inventory y equity capital/common stock son solo algunos de los ejemplos más conocidos de un número muy elevado de términos problemáticos. Por este motivo, resulta imprescindible que el traductor sea consciente de qué términos pueden plantearle dificultades, para prevenir interpretaciones erróneas del mensaje (Mourier 2004: 150).

Parker (2001: 141) afirma que en las traducciones de estados financieros al inglés de empresas de la Unión Europea, la variedad estadounidense prevalece sobre la británica en la selección de terminología (inventories/stocks, sales/turnover, accounts receivable/debtors). Según el autor, solo el término británico reserves prevalece sobre el estadounidense surplus, lo que podría explicarse por la semejanza de la palabra respecto de las correspondientes en las lenguas románicas, junto al hecho de que las NIIF emplean ambos términos por igual. Mourier (2004: 151), sin embargo, considera que la International Accounting Standards Board (IASB) no tiene una política clara 
sobre la variedad de inglés que debe utilizarse en las NIIF y detecta una preferencia por el inglés británico en cuestiones como el uso de 's' frente a la ' $z$ ' (amortisation) y de 'ou' frente a 'o' (labour). Además, mientras el formato del balance propuesto en las NIIF se basa en el modelo estadounidense, el orden de los activos y pasivos en función de su liquidez es propio del modelo británico (Baskerville y Evans 2011b).

Cabe destacar también que la terminología utilizada por la IASB no es estable, sino que está sujeta a modificaciones en las diferentes revisiones periódicas que realiza de las NIIF. Por ejemplo, en 2007, se modificaron los nombres de varias de las cuentas que componen los estados financieros, entre ellas el balance, que pasó de denominarse Balance Sheet a denominarse Statement of Financial Position (Evans 2010: 346), así como de partidas concretas (ej. minority interest pasó a non-controlling interest). Con todo, la propia NIC1 (Presentación de estados financieros) establece en su artículo 10 que la nueva terminología no es obligatoria, sino que las empresas pueden seguir empleando los términos tradicionales. Por otro lado, tampoco en todos los casos el cambio del término en lengua inglesa se traslada a todas las lenguas a las que se traducen las NIIF (Kettunen 2017: 47-48). Ello provoca la coexistencia de diferentes términos con igual significado, lo que puede dificultar la extracción terminológica a través de fuentes normativas y textos paralelos.

Otra cuestión relevante a la que aludíamos en la introducción es la dificultad de identificar un destinatario único que nos permita decantarnos por una estrategia de traducción global determinada. Es poco probable que una empresa encargue traducciones diferentes de sus informes financieros para atraer a posibles inversores de distintos países. Por una parte, esto nos obligará a elegir una variedad de inglés concreta, sea británico, estadounidense o el llamado inglés internacional. Sin embargo, como apunta Biel (2008: 25), la cuestión va más allá de la selección de una variedad, puesto que la existencia de distintos destinatarios multiplicará los sistemas contables de los receptores. Esto supondría un nuevo impedimento para el uso del equivalente funcional como estrategia de traducción prioritaria, dado que lo que se podría considera equivalente en una cultura de llegada podría no serlo en otras.

\section{A qué fuentes recurrir}

Las dificultades que entraña la traducción de textos contables, ya sea de normas de contabilidad o de estados financieros, hacen imprescindible un proceso de documentación reflexionado y exhaustivo. Incluso los traductores con conocimientos avanzados de economía corren el riesgo de cometer errores derivados de una mala selección terminológica o de una interpretación incorrecta del TO. Para orientar de manera satisfactoria este proceso de documentación, desde una perspectiva didáctica, Mayoral (1996) ${ }^{6}$ propone combinar el uso de fuentes léxicas y fuentes textuales (textos multilingües, textos paralelos en la LT tanto originales como traducidos). FuertesOlivera (2003) añade como fuentes textuales los manuales y las monografías, que permiten obtener un conocimiento enciclopédico previo a la traducción. Por su parte, Lobato Patricio, Durán Cruz et al. (2010) sugieren visitar sitios web de organismos internacionales y de las empresas emisoras de los textos originales. Desde la perspectiva profesional, White, Matteson et al. (2008) presentan los recursos utilizados por un grupo de traductores especializados en una pirámide con los diccionarios generales bilingües y monolingües en la parte superior y los recursos de carácter enciclo- 
pédico (revistas especializadas, anuarios, documentos extraídos de internet, enciclopedias) en la base. En los puestos centrales sitúan los diccionarios especializados, seguidos de manuales lingüísticos como gramáticas o diccionarios de uso, y de herramientas desarrolladas de manera específica (diccionarios anotados, glosarios o repositorios de memorias de traducción). No obstante, su análisis se centra sobre todo en los diccionarios, que constituyen, según los resultados de su estudio, la principal fuente de consulta de los traductores encuestados.

Todos los recursos propuestos pueden resultar útiles, a priori, en la traducción de estados financieros. No obstante, se hace necesario constatar su utilidad en la práctica, mediante análisis detallados de su contenido y de su utilidad. Diversos autores (Gerzymish-Arbogast 1989; Dancette 1992; Besomi 2011) se han ocupado del análisis de fuentes de referencia en el ámbito específico de la traducción económica, aunque pocos se han centrado específicamente en la traducción de documentos contables y, de forma más concreta, de estados financieros. Aunque Gallego (2014) realiza un análisis de la utilidad de los recursos terminológicos para la traducción de cuentas anuales de francés a español, el estudio de otro tipo de fuentes ha recibido muy poca atención pese a su importancia en el proceso documental.

No se puede negar que los diccionarios son una de las primeras herramientas que se asocian al trabajo del traductor y que constituyen una fuente valiosa de vocabulario y terminología especializada. Sin embargo, la experiencia en el ámbito de la traducción y de la docencia nos indica que, para ser verdaderamente útil, un diccionario especializado debe ir más allá de proporcionar listados de equivalencias e incluir definiciones en ambas lenguas, contextos de uso, referencias a otras fuentes, recomendaciones, etc. Es cierto que hoy en día existen diccionarios que tienen en cuenta estas necesidades, como es el Diccionario Inglés-Español de Contabilidad (Fuertes-Olivera, Nielsen et al. 2014). Entre sus ventajas principales se pueden mencionar la inclusión de referencias a los contextos de uso de los términos (IRFS, UK, US), definiciones en inglés y español, colocaciones y notas sobre uso. También es de gran utilidad la política aplicada de ofrecer una única equivalencia por lema, basada en el principio de evitar el estrés que provoca en el usuario obtener más información de la que necesita (Fuertes-Olivera 2011: 107).

Con todo, como sucede con todas las obras lexicográficas, su extensión es limitada, por lo que no cubre todos los términos que se pueden encontrar en un documento contable, en especial los términos compuestos utilizados para denominar subcuentas dentro de los estados financieros. Por ejemplo, la búsqueda de las subpartidas marketable equity securities y marketable debt securities del Statement of Financial Condition prototípico del sistema contable estadounidense nos remite en ambos casos al hiperónimo marketable securities para el cual se ofrece la equivalencia española títulos negociables, junto con una indicación de que en el balance español se denominan inversiones financieras a corto plazo. No obstante, en el Statement of Financial Condition, las inversiones financieras a corto plazo se denominan short term investments e incluyen efectivamente las dos subpartidas anteriores. Por su parte, la búsqueda de debt securities nos proporciona el equivalente títulos de deuda, mientras que la de equity securities nos lleva a título de patrimonio. A partir de esa información, un traductor con conocimientos de contabilidad suficientes para moverse con seguridad en el entramado terminológico que esta supone, podría optar por los términos títulos de deuda negociables y títulos de patrimonio negociables. Sin 
embargo, con el uso exclusivo del diccionario no podría llegar al término exacto con el que se denominan las subpartidas equivalentes en el balance español, a saber, instrumentos de patrimonio y valores representativos de deuda. Para llegar a estos términos, necesitará recurrir a otro tipo de fuentes.

Otra limitación de los diccionarios, en especial los diccionarios impresos, es su incapacidad para mantenerse al día de los cambios en la terminología que se producen en la lengua económica. Aunque esto es relevante en el caso de los neologismos, lo es más aún en los casos de cambio semántico, el mecanismo más habitual para el desarrollo terminológico en el ámbito de la economía (Crystal 1987). Es más fácil detectar la acuñación de un término nuevo que detectar que un término ha modificado su significado (ej. material entendido como sustancial en lugar de como relativo a la materia) o que un término ha dejado de utilizarse en favor de otro (ej. corriente en lugar de circulante). De nuevo en estos casos será fundamental el uso de fuentes alternativas.

En las páginas siguientes, valoraremos la utilidad de las fuentes normativas y de los textos paralelos en la traducción de estados financieros. Para ello, partiremos de una selección de términos habituales de la redacción en español y en inglés para intentar determinar si las fuentes nos permiten tomar una decisión segura sobre la forma más adecuada para traducirlos.

\subsection{Fuentes normativas del ámbito de la contabilidad}

Consideramos fuentes normativas las resoluciones dictadas por los órganos legislativos y judiciales: en el caso de la contabilidad, todas aquellas que tienen que ver con la formulación de estados financieros por parte de las sociedades mercantiles. Tienen como ventaja, sobre otro tipo de fuentes, que suelen ser de fácil acceso, a través bien de fuentes primarias como los boletines oficiales, bien de fuentes secundarias como bases de datos jurídicas, repertorios legislativos o sitios web de entidades oficiales.

Como vimos en el apartado 2, Nobes y Stadler (2017: 17) recomiendan que los traductores de estados financieros recurran a las NIIF como fuente de referencia para garantizar un uso uniforme de la terminología contable. Consideran que, de esta manera, en las traducciones de otras lenguas al inglés, se podrán corregir los errores terminológicos y las faltas de equivalencia cometidos en la redacción de los originales. Aunque su recomendación no carece de sentido, requiere por parte del traductor un cierto conocimiento de las NIIF, así como de sus procesos de adopción, revisión y traducción.

\subsubsection{Adopción de las NIIF}

Hasta la adopción de las NIIF acordada por la UE para las empresas cotizadas, cada estado se regía por sus propias leyes contables. Si bien los principios básicos eran semejantes, existían diferencias importantes derivadas del hecho de estar generadas en estados con sistemas contables distintos. Así, mientras en los países de tradición continental, la práctica contable se basaba principalmente en lo establecido por la legislación estatal, como el PGC y la normativa fiscal española o el Handelgesetzbuch $(\underline{H G B})$ en Alemania, en los países más próximos al derecho común las normas contables solían emanar de normativas elaboradas en el sector privado, como los Statements of Accounting Standard Practice publicados por el Institute of Chartered Accounts in England and Wales (ICAEW). 
El Reglamento CE 1606/2002 del Parlamento Europeo y del Consejo estableció la obligación de todas las empresas nacionales cotizadas de los estados miembros de la UE y del espacio económico europeo de utilizar las NIIF-UE ${ }^{7}$ en la formulación de sus estados financieros a partir de enero de 2005. Esto supuso una cierta armonización de las políticas contables dentro de la Unión Europea, aunque no garantizó una convergencia total, ya que cada Estado es libre de decidir cómo deben comportarse en materia contable el resto de las empresas. Ya nos referimos en la introducción a la diversidad de normas aplicables a las empresas en España. Por su parte, en Reino Unido, las empresas cotizadas deben utilizar de manera obligatoria las NIIF-UE para sus informes consolidados, aunque no para los individuales. El resto de las empresas pueden optar por formular sus informes según la norma FRS102 (Financial reporting standard applicable in the UK and Republic of Ireland), una versión adaptada de las NIIF para pymes. Además, las empresas que cumplen los requisitos pueden acogerse a la FRS101 (Reduced Disclosure Framework). Este patrón de alternancia de normas es la práctica habitual en casi todos los estados de la UE.

Fuera de la UE, son mayoría los estados que han adoptado las NIIF como marco de referencia para sus estados financieros, con alcances diferentes. Las principales excepciones son Estados Unidos, que pese a algunos proyectos de convergencia, ha interrumpido por el momento los planes de cambiar sus prácticas contables (si bien es cierto que las NIIF se derivan parcialmente de los US GAAP [US Generally Accepted Accounting Principles]); Japón, que permite el uso de las NIIF pero no lo exige; y China, que tiene prevista su adopción en un futuro indeterminado ${ }^{8}$.

\subsubsection{Traducción de las NIIF}

La progresiva adopción de las NIIF, tanto en la UE como en muchos otros estados, ha ido acompañada de un proceso de traducción de las normas a las lenguas oficiales de los estados adoptantes. Hasta 1997, cada estado se encargaba de elaborar sus propias traducciones. Ello provocaba la coexistencia de diferentes versiones en una misma lengua, lo que dificultaba la armonización. Por ese motivo, desde 1997, la Fundación IFRS se encarga de regular el proceso, mediante una política de traducción que describe los pasos que deben seguirse durante el proceso de trasvase y los principios básicos a los que deben atenerse las traducciones. El proceso incluye la revisión por parte de un comité de expertos formado por hablantes nativos de las lenguas implicadas.

En la UE, el Reglamento UE 1606/2002, en su artículo 3.4., establecía que se publicarían versiones de las NIIF-UE en todas las lenguas oficiales de la UE. Hasta 2009, las traducciones eran realizadas en parte por la Dirección General de Traducción (DGT) de la Comisión y en parte por la Fundación IFRS en nombre de la UE, con el apoyo de los gobiernos estatales y de la DGT. Desde ese año, las traducciones de las NIIF-UE consideradas como oficiales por la Unión Europea son responsabilidad exclusiva de la DGT. En algunos estados, como Finlandia y Alemania, existe coordinación con el equipo de traducción y revisión que elabora las versiones NIIF para la Fundación IFRS pero en otros estados, como España, Italia, Suecia, Francia y Portugal, las traducciones se realizan de forma independiente (Kettunen 2017: 43, 52). Esto supone la existencia de dos versiones traducidas de las NIIF, las de la Fundación IFRS y las de la DGT, entre las que se pueden encontrar discrepancias terminológicas. Mientras en las primeras se intenta elegir terminología que sea 
aplicable a los países de Latino-América y a Canadá, por ejemplo, las traducciones de la DGT se basan únicamente en las variedades lingüísticas de los países de la Unión (Evans, Baskerville et al. 2015: 4). Así, en la NIC 12 (Impuesto sobre las ganancias), el término inglés accounting profit se traduce en la versión de la UE como resultado contable frente al término ganancia contable empleado en la versión de la Fundación IFRS. Es importante que el traductor tenga en cuenta estas posibles discrepancias cuando seleccione el documento normativo que utilizará como referencia.

Otro problema en el uso de las NIIF como fuente de extracción terminológica podría ser la falta de correspondencia exacta en la terminología empleada de unas lenguas a otras. Son claros ejemplos de esto el término inglés cost, que puede referirse a gasto o coste en función del contexto (igual diferencia existe en la lengua finesa, Kettunen 2017: 47) o los términos depreciation y amortisation (véase apartado 2.2). Esta falta de correspondencia provoca problemas en las traducciones de las propias NIIF, como reflejan los siguientes ejemplos:

1) Depreciation (Amortisation) is the systematic allocation of the depreciable amount of an asset over its useful life.*

* In the case of an intangible asset, the term 'amortisation' is generally used instead of 'depreciation'. The two terms have the same meaning.

a) Amortización es la distribución sistemática del importe amortizable de un activo a lo largo de su vida útil (1).

(1) En el caso de los activos intangibles, se suele usar el término «amortización» en lugar de depreciación. Ambos términos tienen el mismo significado.

(NIC 36 - NIIF-UE)

2) Although this Standard uses the terms 'other comprehensive income', 'profit or loss' and 'total comprehensive income', an entity may use other terms to describe the totals, as long as the meaning is clear. For example, an entity may use the term 'net income' to describe profit or loss.

a) Aunque esta Norma utiliza los términos "otro resultado global", "resultado" y "resultado global total", una entidad puede utilizar otros términos para denominar los totales, siempre que el significado quede claro. Por ejemplo, una entidad puede utilizar el término "resultado neto" para denominar al resultado.

(NIC 1 - NIIF-IFRS)

Por otra parte, dado que la adopción de las NIIF no ha sido generalizada en todas las empresas de un estado, se dan situaciones en que un mismo término puede seguir utilizándose con significados diferentes según se utilicen las NIIF o la normativa estatal. Callao, Jarne et al. (2007: 159) constataron que, pese a emplear terminología igual o semejante, los resultados de elaborar los estados financieros de una empresa según el PGC o según las NIIF divergían para varias de las partidas comunes del balance y de la cuenta de resultados. Estas divergencias se debían a diferencias en el contenido semántico de la terminología empleada. Así, la partida inmovilizado intangible, de acuerdo con el PGC, incluye, entre otros elementos, los gastos de I+D. La NIC 38 (Activos intangibles), por el contrario, obliga a cargar a resultados los gastos de investigación, mientras que los de desarrollo pueden reconocerse como intangible cuando se dan ciertas condiciones. Como consecuencia, el término inmovilizado 
intangible tendrá diferente alcance semántico cuando se utilice en un informe financiero elaborado según el PGC que cuando se utilice en uno elaborado según las NIIF. Emplear la terminología NIIF para traducir al inglés unas cuentas PGC o viceversa puede llevar a los potenciales inversores a interpretar que los componentes de las partidas valoradas son exactamente los mismos.

\subsection{Textos paralelos y corpus paralelos de estados financieros}

Sánchez (2002) define textos paralelos como «textos originales en la lengua de partida o en la de llegada sobre el mismo tema y cuya función es similar o equivalente a la de la traducción». Baker (1995: 230), por su parte, define corpus paralelo como aquel que está formado por textos originales en una lengua y sus traducciones a otra. En el primer caso englobaríamos por tanto los estados financieros emitidos por empresas en su lengua original, mientras que en el segundo nos referimos a estados financieros en lengua original confrontados con sus traducciones.

La facilidad de acceso a este tipo de fuentes difiere según se trate de originales o traducciones y, en el caso de estas últimas, de su finalidad. No suele ser difícil acceder a estados financieros originales, ya que se trata de documentos que han de depositarse ante la Administración y que, en muchos casos, las propias empresas publican en sus sitios web. Por lo que se refiere a las traducciones, tampoco resulta difícil el acceso cuando se trata de versiones hacia el inglés elaboradas con el objetivo de atraer inversores. Por el contrario, resulta complicado acceder a traducciones de estados financieros anglosajones traducidos a otras lenguas, al menos en el caso del español. Esto se debe a que se trata de una práctica poco habitual, como ya se indicó en el apartado 2. Por otro lado, las pocas traducciones que se realizan suelen tener como destinatarios personas físicas y jurídicas concretas y no están destinadas a su publicación en el sitio web de una empresa para su acceso público por parte de los terceros interesados.

Un factor que el traductor no debe olvidar al utilizar estados financieros como fuentes de extracción terminológica es el hecho de que las versiones traducidas de las NIIF no siempre están disponibles con igual rapidez en todas las lenguas. Por este motivo, los responsables de elaborar las cuentas anuales a veces utilizan la versión inglesa de las normas (Huerta, Petrides et al. 2013). Esto ocasiona una variabilidad aún mayor de la terminología empleada, ya que en estos casos se suelen calcar de manera literal los términos ingleses, sin realizar un ejercicio de reflexión sobre la pertinencia o no de utilizar dichos calcos. Por otra parte, dado que los estados financieros son modelos estandarizados que las empresas actualizan anualmente con nuevos datos, pero que no se redactan de nuevo cada año, en ocasiones no se introducen los cambios terminológicos en todas las secciones del informe, lo que puede provocar que en un mismo documento se utilicen distintos términos con el mismo significado. En la traducción estos problemas se perpetúan, ya que los términos diferentes podrán ser vertidos a la otra lengua de formas también diferentes, por lo que el traductor deberá tener aún más cautela cuanto utilice corpus paralelos como fuente de extracción. 


\section{Análisis práctico de los recursos}

En el apartado anterior se presentan los recursos disponibles para la traducción financiera de una manera teórica, basada principalmente en la revisión bibliográfica. Sin embargo, para valorar verdaderamente su utilidad durante el proceso de traducción, hemos considerado necesario realizar un análisis práctico, basado en la explotación terminológica a partir de varias tareas de búsqueda y comparación de términos seleccionados.

\subsection{Tareas realizadas}

En primer lugar, se partió de un glosario elaborado por Ramin y Reinman (2013), que recoge alrededor de 350 términos especializados extraídos de la versión inglesa de las NIIF junto con sus definiciones, y se recuperaron esos mismos términos y definiciones de las versiones españolas realizadas por la Fundación IRFS y por la DGT. Se buscaba de esta forma comprobar las diferencias de uso terminológico entre estas dos versiones.

A continuación, se seleccionaron varios de los términos mencionados en los artículos de la revisión bibliográfica, a los que hemos hecho alusión en los apartados 2 y 3 (amortisation, fair value, statement of financial position, measurement, revenue, receivables...) para analizar su uso en inglés y en español tanto en la normativa internacional como en cuentas reales de empresas. Para valorar su uso en la normativa internacional se compararon las versiones inglesa y española de las NIIF originales de la Fundación IFRS y las mismas versiones de las NIIF-UE. Para el análisis en cuentas de empresas, se compiló un corpus monolingüe de originales en lengua inglesa formado por 44 estados financieros de empresas estadounidenses (22) y británicas (22) y un corpus paralelo formado por la versión original española y la traducción al inglés de los estados financieros de las empresas del IBEX-35.

Por último, se realizó un vaciado terminológico del Estado de Situación Financiera de las 22 empresas estadounidenses, que se comparó con el modelo de Balance propuesto en las US GAAP. Después, se buscaron posibles equivalentes de las partidas en el modelo de balance del PGC de 2007 y en los componentes del balance propuestos en el art. 54 de la NIC1 (art. 68 de la NIC1-UE), para finalmente contrastar los resultados con los balances de las empresas británicas y españolas.

\subsection{Resultados}

A continuación, se presentan de forma sucinta los resultados más relevantes del análisis anterior. La presentación de los resultados completos excede los límites de este artículo y será por lo tanto objeto de un nuevo trabajo.

En términos generales, aunque se puede confirmar la existencia de diferencias entre la terminología NIIF-IFRS y NIIF-UE, estas no son tan habituales como la bibliografía parece indicar y suelen consistir en una preferencia en la primera versión por términos más calcados del original inglés, por ejemplo:

- beneficios/prestaciones,

- costo/coste,

- tasa de interés/tipo de interés,

- inventarios/existencias, 
- disposición/enajenación,

- operación/explotación,

- negocio en marchalempresa en funcionamiento,

- gobierno/administraciones públicas,

- controladoraldominante,

- propiedades/inmuebles,

- terminación/cese.

Las diferencias en términos más específicos son prácticamente inexistentes y se han ido reduciendo aún más en las últimas revisiones de las normas, como es el caso de los términos Estado de situación financiera y Estado de resultado global, no utilizados en las primeras versiones de las NIIF-UE.

El análisis de los corpus revela una mayor uniformidad en los estados financieros de las empresas británicas y españolas que en las estadounidenses, sobre todo en las partidas incluidas en el balance, que en las empresas de Estados Unidos difieren considerablemente de unas a otras. Esto dificulta sistematizar las búsquedas de terminología a partir del corpus y encontrar posibles equivalentes en los modelos de balance españoles o internacionales.

Por lo que se refiere al uso de la terminología en los diferentes corpus, se aprecia una variabilidad considerable en las opciones terminológicas de las empresas, aunque esta es menos habitual en los estados originales en inglés y en español que en las traducciones al inglés. Lo que más problemas supone para la explotación de los textos paralelos como recurso terminológico es el hecho de que en muchos informes se detecta el uso de diferentes opciones terminológicas para un mismo concepto, algo que sucede en los nombres de los propios estados (como es el caso del Estado de Situación Financiera, que también aparece denominado Balance en muchos informes) y también con términos más generales (como valoración y medición o share premium y additional paid-in capital). No obstante, esta práctica se detecta con mayor frecuencia en los estados financieros en español mientras que es poco frecuente en los elaborados por las empresas británicas. Por ejemplo, todas las empresas españolas utilizan el término inmovilizado material en sus informes. Sin embargo, cuatro de ellas utilizan también el término literal propiedad, planta y equipo, que es el que aparece en la versión española de la Fundación IRFS. Por su parte, solo diez empresas estadounidenses utilizan el término property, plant and equipment, mientras dos de ellas utilizan fixed assets y una office facilities y el resto de los balances no incluían esta partida. Por último, todas las empresas británicas, a excepción de una, utilizaban property, plant and equipment.

En las traducciones al inglés de los estados financieros del IBEX-35 se detectó un empleo parejo de las variedades británica y estadounidense, si bien hasta en 13 informes se empleaban elementos de ambas, como el uso alternado de amortisation y amortization. Asimismo, se identificaron usos en el mismo documento y con el mismo valor de creditors y receivables, de minority interest y non-controlling interest, y de turnover y revenue, entre otros. En algunos casos se detectaron prácticas de traducción cuestionables, como el uso de depreciation para hacer referencia a la amortización del activo intangible, que sin llegar a ser un error podría resultar extraño para un receptor experto. Por otra parte, cuando se traducen al inglés partidas propias del sistema contable español, como Hacienda pública deudora o Hacienda pública acreedora, las opciones terminológicas son mucho más variadas, 
siempre con tendencia hacia la neutralización del término, más que hacia el uso de un equivalente funcional en otros sistemas.

Finalmente, resultó de especial interés el estudio de las expresiones true and fair view, fair presentation y present fairly. La noción true and fair view no es propia de las NIIF, sino que se introduce en la legislación contable de la UE a través de la cuarta y séptima directivas. Sí se recoge en la ISA 700, norma internacional de auditoría, que permite optar entre present fairly y give a true and fair view en el informe. La NIC1, por su parte, requiere que los estados financieros presenten fielmente/present fairly la situación financiera de la empresa y de dicha obligación se deriva el concepto fair presentation que en la versión española de la NIC1 se tradujo como imagen fiel, de manera que coincide con el término utilizado para true and fair view. Por este motivo, todas las empresas españolas emplean en sus cuentas la expresión imagen fiel. No obstante, en las traducciones al inglés, solo 20 utilizan true and fair view, mientras que en otros casos se utiliza la alternativa fair presentation (4), o incluso traducciones inapropiadas como true picture (2) o true image (4).

\section{Conclusiones}

La revisión bibliográfica y el análisis de fuentes normativas y textuales nos muestran un ámbito de la traducción contable dominado por dos géneros: el de las normas contables, en las que la traducción se produce fundamentalmente del inglés hacia otras lenguas, y el de los estados financieros, en los que predomina la traducción de otras lenguas hacia el inglés. Pese a tratarse de géneros diferentes, la bibliografía refleja la estrecha vinculación que existe entre ellos y el importante papel que la traducción desempeña en la inteligibilidad de ambos. Ante este hecho, resulta relevante referirse al grado de competencia que debe tener un traductor para poder llegar a traducir un informe financiero de manera satisfactoria en un plazo de tiempo asequible. Dada la diversidad de fuentes normativas y textuales disponibles y la variación en el uso de la terminología, un traductor sin conocimientos especializados de contabilidad y sin una buena sub-competencia instrumental requeriría mucho tiempo para procesar toda la información necesaria para tomar las decisiones de traducción más apropiadas. Muchas veces, no se dispone del tiempo necesario, por lo que se tiende a priorizar el uso de las fuentes más productivas con una inversión de tiempo menor, como son los diccionarios, y a utilizar las fuentes alternativas para obtener una visión de conjunto del texto y de la temática y para resolver dudas terminológicas puntuales no resueltas por los diccionarios y cuestiones asociadas al contenido, como las ambigüedades. No obstante, la revisión bibliográfica y el análisis de fuentes demuestran que esta práctica puede provocar errores en las traducciones, tanto de las normas como de los estados.

A la vista de esta situación, cabe proponer unas pautas de actuación, basadas en los resultados de nuestro análisis, que ayuden al traductor a gestionar las fuentes disponibles de la forma más productiva posible. Como punto de partida, sería deseable determinar cuál es la normativa de referencia de las cuentas del TO y qué sistema contable servirá de referencia al receptor del TM para interpretar el texto. El análisis de las fuentes normativas indica que las versiones inglesa y española de las NIIF pueden resultar una buena herramienta de extracción terminológica sobre la que sustentar las decisiones de traducción. En los casos de traducción directa al español, 
las NIIF serán la normativa de referencia de un gran número de países. Incluso si se trata de estados financieros procedentes de Estados Unidos, el origen común de las NIIF y las GAAP garantizan una cierta armonización. Por su parte, el origen del destinatario del TM determinará si se ha de optar por la versión española de la Fundación IRFS o de la DGT, si bien, el análisis de las fuentes indica que las diferencias entre ellas no son tan determinantes como para que la elección de una u otra ponga en peligro la equivalencia funcional del TM en su conjunto. En los casos de traducción inversa del español al inglés, la normativa de referencia del TO variaría en función del tamaño de la empresa, aunque lo más habitual es que sean las empresas cotizadas las que traduzcan sus cuentas. El sistema de referencia de llegada puede reducirse a dos, las US GAAP para receptores de Estados Unidos o las NIIF para receptores de otros países. No obstante, dado que resulta difícil discriminar si las cuentas estarán destinadas a lectores de un único estado, cuando la traducción se realice solo con fines informativos, lo más recomendable es optar por las NIIF como modelo de referencia, que garantiza un mayor alcance del TM, además de una mayor similitud al texto de partida español. Recordemos que incluso si la empresa elabora sus cuentas con arreglo al PGC, las similitudes serán mayores con las NIIF que con las US GAAP, ya que la normativa estatal ha ido adaptándose a las primeras desde su adopción como normas de referencia para empresas cotizadas. Solo en aquellos casos en los que la traducción constituya un paso intermedio hacia una consolidación de cuentas con una matriz de un país concreto, podría ser recomendable utilizar la normativa de ese país como fuente de referencia.

Por lo que se refiere a los textos paralelos, el uso de informes financieros de empresas estadounidenses parece poco productivo para la traducción al inglés de informes financieros de empresas españolas, por presentar diferencias importantes en las opciones terminológicas respecto a las NIIF. Por el contrario, las memorias de las empresas británicas se revelan como una buena fuente de extracción. Su uso resulta más recomendable, en términos generales, que el uso de traducciones al inglés de estados financieros en español, ya que el hecho de estar formuladas a partir de la normativa original inglesa les confiere una mayor uniformidad, lo que reduce el tiempo de procesamiento necesario.

Desde una perspectiva didáctica, resulta indispensable que los futuros traductores aprendan a utilizar de manera crítica las fuentes normativas y los textos y corpus paralelos desde el principio de su formación, en un entorno que favorezca el trabajo documental integral en lugar de la búsqueda término a término. El análisis reflexivo de los recursos les permitirá concluir por sí mismos que, a corto plazo, un mayor número de recursos no significa necesariamente una reducción del tiempo necesario para realizar la traducción, sino una capacidad de decisión mejor sustentada. A largo plazo, no obstante, el dominio de la sub-competencia instrumental les permitirá tomar decisiones de traducción con mayor seguridad y con una inversión de tiempo menor.

\section{NOTAS}

1. En inglés, International Financial Reporting Standards (IFRS). Las NIIF incluyen las NIC, las SIC, las NIIF y las CINIIF (Interpretaciones de las NIIF). Véase anexo 1.

2. NIIF-UE se refiere a la versión de las normas aprobada por la Unión Europea, parcialmente modificadas con respecto a las originales. 
3. Hellmann, Andreas, Perera, Hector y Patel, Chris (2010): Equivalence of IFrS across Languages: translation issues from English to German, sin publicar. APIRA 2010: Sixth Asia Pacific Interdisciplinary Research in Accounting Conference, Sidney, 11-13 de julio de 2010.

4. Nobes, Christopher W. y StAdler, Christian (2017): Impaired translations: IFRS from English and annual reports into English. Eufin 2017: 13th Workshop on European Financial Reporting, Florencia, 31 de agosto a 1 de septiembre de 2017.

5. Tsakumis, George T., Campbell, David R. y Doupnik, Timothy S. (1 de febrero de 2009): IFRS: Beyond the standards. Journal of Accountancy. Consultado el 18 de noviembre de 2018, <https:// www.journalofaccountancy.com/issues/2009/feb/ifrsbeyondthestandards.html>.

6. MAYORAL, Roberto (1996): La traducción comercial: estrategias de traducción en razón a la disponibilidad de las fuentes de referencia terminológicas y textuales, sin publicar. V Curso Superior de Traducción Inglés/Español, Valladolid, 18 de abril de 1996.

7. El Reglamento CE 1725/2003 recogía la versión NIIF-UE para su aplicación en la Unión Europea y fue modificado en 18 ocasiones hasta ser derogado en noviembre de 2008 por el Reglamento CE 1126/2008. En este se recoge la versión actual de las NIIF-UE y desde su publicación ha sido objeto de 51 enmiendas dirigidas a derogar, actualizar e incorporar las normas.

8. IFRS FOUNDATION (Actualizado: 25 de abril de 2019): Use of IFRS Standards by Jurisdiction. IFRS. Consultado el 5 de junio de 2019, <http://www.ifrs.org/use-around-the-world/use-of-ifrs-standards-by-jurisdiction/>.

\section{REFERENCIAS BIBLIOGRÁFICAS}

AlCalde, Elena (2013): La traducción del balance de situación: análisis de dificultades y propuesta de actividades mediante un estudio de corpus. In: Emilio OrTEga, ed. Translating Culture. Granada: Comares, 437-448.

Alexander, David y Nobes, Christopher W. (2001): Financial Accounting: An International Introduction. Harlow: Pearson.

Archer, Simon y McLeay, Stuart (1991): Issues in Transnational Financial Reporting: A Linguistic Analysis. Journal of Multilingual and Multicultural Development. 12(5):347-361.

BAKER, Mona (1995): Corpora in translation studies. An overview and some suggestions for future research. Target. 7(2):223-243.

BASKeRVILle, Rachel y EvAns, Lisa (2011a): The darkening glass: Issues for translation of IFRS. Edimburgo: The Institute of Chartered Accountants of Scotland.

BASKerville, Rachel y Evans, Lisa (2011b): Situating IFRS translation in the EU within speed wobbles of convergence. SSRN Electronic Journal. Consultado el 15 de abril de 2018, <http:// dx.doi.org/10.2139/ssrn.1913448>.

Besomi, Daniele (2011): Crises and Cycles in Economic Dictionaries and Encyclopaedias. Londres/ Nueva York: Routledge.

BiEL, Łucja (2008): Legal terminology in translation practice: Dictionaries, googling or discussion forums? SKASE Journal of Translation and Interpretation. 3(1):22-38.

CAllao, Susana, JARne, José y LAÍnEz, José A. (2007): Adoption of IFRS in Spain: Effect on the comparability and relevance of financial reporting. International Accounting Auditing \& Taxation. 16:148-178.

Campbell, David, Beck, Cornelia A. y Shrives, Philip J. (2005): A note on comparative language interrogation for content analysis: The example of English vs. German. British Accounting Review. 37(3):339-350.

CARMona, Alejandro (2012): El balance de situación en el proceso de internacionalización empresarial: Aplicación de un modelo traductológico integrador para su análisis y propuesta de traducción (español-francés). Tesis de doctorado, sin publicar. Granada: Universidad de Granada.

Clark, Peter (1994): European Financial Reporting. Luxemburgo/Londres: Routledge.

Courtis, John K. y Hassan, Salleh (2002): Reading ease of bilingual annual reports. Journal of Business Communication. 39(4):394-413.

Crystal, David (1987): The Cambridge Encyclopedia of Language. Cambridge: Cambridge University Press. 
DAhlgren, Jörgen y NiLsson, Sven-Arne (2012): Can translations achieve comparability? The case of translating IFRSs into Swedish. Accounting in Europe. 9(1):39-59.

DANCETTE, Jeanne (1992): La complexité de la langue économique et commerciale au Québec, les défis du traducteur. Terminologie et Traduction. 2(3):197-210.

Doupnik, Timothy S. y RichteR, Martin (2003): Interpretation of Uncertainty Expressions: A Cross-National Study. Accounting, Organizations and Society. 28(1):15-35.

Evans, Lisa (2004): Language, translation and the problem of international accounting communication. Accounting, Auditing and Accountability Journal. 7(2):210-248.

Evans, Lisa (2010): Observations on the Changing Language of Accounting. Accounting History. 15(4):439-462.

Evans, Lisa, BASkerville, Rachel y Nara, Katariina (2015): Colliding Worlds: Issues Relating to Language Translation in Accounting and Some Lessons from Other Disciplines. Abacus. 51(1):1-36.

Fuertes-Olivera, Pedro A. (2003): La relevancia de la labor de documentación en la traducción económica. In: Pilar Castaño, Tobias T. Klinge, Luis Pérez González et al., eds. La traducción y la interpretación en un entorno global. Villaviciosa de Odón: Universidad Europea de Madrid.

Fuertes-Olivera, Pedro A. (2011): Equivalent Selection in Specialized e-Lexicography: A Case Study with Spanish Accounting Terms. Lexikos. 21:95-119.

Fuertes-Olivera, Pedro A. Nielsen, Sandro y Bergenholtz, Henning (2014): The 'Diccionario Inglés-Español de Contabilidad: Traducción' and the 'Diccionario Inglés-Español de Contabilidad: Traducción de Frases Hechas y Expresiones': Two Specialised Dictionaries for Translating Terms and Collocations. MonTI. 6:91-114.

Gallego, Daniel (2014): Evaluación de recursos terminológicos en el ámbito contable. MonTI. 6:141-166.

GARCíA, Marta (2017): A review of the concept of functional equivalent in translation: business entity types in Spain and in the United States. Perspectives. 25(3):378-396.

García, Marta y Veiga, Maite (2014): Guided Inquiry and Project-Based Learning in the field of specialised translation: a description of two learning experiences. Perspectives. 23(1):107123.

Gerzymish-Arbogast, Heidrun (1989): The Role of Sense Relations in Translating Vague Business and Economic Texts. In: Mary SNell-Hornby y Ester PöHL, eds. Translation and Lexicography: Papers read at the Euralex Colloquium. Ámsterdam/Filadelfia: John Benjamins, 187-196.

Holthoff, Gero, Hoos, Florian y Weissenberger, Barbara E. (2015): Are We Lost in Translation? The Impact of Using Translated IFRS on Decision-Making. Accounting in Europe. 12(1):107-125.

Huerta, Esperanza, Petrides, Yanira y Braun, Gary P. (2013): Translation of IFRS: Language as a barrier to comparability. Research in Accounting Regulation. 25:1-12.

Jeanjean, Thomas, Lesage, Cédric y Stolowy, Hervé (2010): Why do you speak English (in your annual report)? The International Journal of Accounting. 45(2):200-223.

Johanson, Jan y Vahlne, Jan-Erik (1977): The Internationalization Process of the Firm: A model of knowledge development and increasing foreign market commitments. Journal of International Business Studies. 8(1):23-32.

Kettunen, Jaana (2017): Interlingual translation of the International Financial Reporting Standards as institutional work. Accounting, Organizations and Society. 56:38-54.

LAÍNEZ, José A. (1993): Comparabilidad internacional de la información financiera: análisis y posición de la normativa española. Madrid: Instituto de Contabilidad y Auditoría de Cuentas.

Lobato Patricio, Julia, Durán Cruz, Natalia y Morón Sánchez, Carmen María (2010): La traducción francés-español de textos económicos de divulgación desde una perspectiva didáctica. AdVersuS. 7:73-106.

Mourier, Lisa (2004): Communicating financial reporting across continents. Hermes, Journal of Linguistics. 32:145-165. 
Nobes, Christopher W. (2015): Accounting for capital: the evolution of an idea. Accounting and Business Research. 45(4):413-441.

PARKer, Robert H. (2001): European languages of account. European Accounting Review. 1:133147.

Pizarro SÁnchez, Isabel (2000): El registro económico de la lengua inglesa: análisis del género "memorias anuales" y de sus traducciones al inglés. Tesis de doctorado, sin publicar. Valladolid: Universidad de Valladolid.

Ramin, Kurt P. y Reinman, Cornelis A. (2013): IFRS and XBRL: How to Improve Business Reporting Through Technology and Object Tracking. Chichester: John Wiley \& Sons.

SÁnchez, Pilar (2002): Aplicaciones de la Lingüística de Corpus a la práctica de la TraducciónComplemento de la Traducción Asistida por Ordenador. Terminologie et Traduction. 2:84-106.

Smith, Sylvia A. (1995): Culture clash: Anglo-American case law and German civil law in translation. In: Marshall Morris, ed. Translation and the Law. Ámsterdam/Filadelfia: John Benjamins, 179-197.

White, Marilyn Domas, Matteson, Miriam y Abels, Eileen G. (2008): Beyond dictionaries: Understanding information behavior of professional translators. Journal of Documentation. 64(4):576-601.

Zeff, Stephen A. (2007): Some Obstacles to Global Financial Reporting Comparability and Convergence at a High Level of Quality. The British Accounting Review. 39:290-302.

\section{ANEXOS}

Anexo 1: Términos y siglas

\begin{tabular}{|c|l|l|l|}
\hline \multicolumn{2}{|c|}{ Español } & \multicolumn{2}{c|}{ Inglés } \\
\hline Sigla & \multicolumn{1}{|c|}{ Término } & Sigla & \multicolumn{1}{c|}{ Término } \\
\hline NIIF & $\begin{array}{l}\text { Normas Internacionales de } \\
\text { Información Financiera }\end{array}$ & IFRS & $\begin{array}{l}\text { International Financial Reporting } \\
\text { Standards }\end{array}$ \\
\hline CINIIF & Comité de Interpretaciones de las NIIF & IFRIC & $\begin{array}{l}\text { International Financial Reporting } \\
\text { Interpretations Committee }\end{array}$ \\
\hline NIC & Norma Internacional de Contabilidad & IAS & International Accounting Standards \\
\hline SIC & $\begin{array}{l}\text { Comité de Interpretaciones de las } \\
\text { Normas Internacionales de } \\
\text { Contabilidad }\end{array}$ & SIC & Standing Interpretations Committee \\
\hline
\end{tabular}

\section{Anexo 2: Reglamentos, decretos, directivas, circulares y resoluciones}

BANCO DE EsPaña (30 de diciembre de 2004): Circular 4/2004, de 22 de diciembre, a entidades de crédito, sobre normas de información financiera pública y reservada y modelos de estados financieros. Boletín Oficial del Estado. 314:42410-42508. Consultado el 3 de marzo de 2019, <https://www.boe.es/eli/es/cir/2004/12/22/4>.

BAnCo DE España (10 de diciembre de 2008): Circular 6/2008, de 26 de noviembre, del Banco de España, a entidades de crédito, de modificación de la Circular 4/2004, de 22 de diciembre, sobre normas de información financiera pública y reservada, y modelos de estados financieros. Boletín Oficial del Estado. 297:49385-49456. Consultado el 4 de marzo de 2019, $<$ https://www.boe.es/eli/es/cir/2008/11/26/6(b)>.

Comisión Europea (29 de septiembre de 2003): Reglamento (CE) nº 1725/2003 de la Comisión, de 29 de septiembre de 2003, por el que se adoptan determinadas Normas Internacionales de Contabilidad de conformidad con el Reglamento (CE) n 1606/2002 del Parlamento Europeo y del Consejo. Diario Oficial de la Unión Europea. L261:1-420. Consultado el 3 de junio de 2019, <https://eur-lex.europa.eu/legal-content/ES/ALL/?uri=CELEX:32003R1725>. 
Comisión Europea (3 de noviembre de 2008): Reglamento (CE) n o 1126/2008 de la Comisión, de 3 de noviembre de 2008, por el que se adoptan determinadas normas internacionales de contabilidad de conformidad con el Reglamento (CE) n o 1606/2002 del Parlamento Europeo y del Consejo. Diario Oficial de la Unión Europea. L320:1-481. Consultado el 3 de junio de 2019, <https://eur-lex.europa.eu/legal-content/es/ALL/?uri=CELEX:32008R1126>.

Consejo de la Unión Europea (25 de julio de 1978): Cuarta Directiva 78/660/CEE del Consejo, de 25 de julio de 1978, basada en la letra g) del apartado 3 del artículo 54 del Tratado y relativa a las cuentas anuales de determinadas formas de sociedad. Diario Oficial de la Unión Europea. L222:0011-0031. Consultado el 24 de junio de 2019, <https://eur-lex.europa.eu/ legal-content/ES/ALL/?uri=CELEX:31978L0660>.

Consejo de la Unión Europea (30 de diciembre de 1989): Undécima Directiva 89/666/CEE del Consejo, de 21 de diciembre de 1989, relativa a la publicidad de las sucursales constituidas en un Estado Miembro por determinadas formas de sociedades sometidas al Derecho de otro Estado. Diario Oficial de la Unión Europea. 395:0036-0039. Consultado el 4 de marzo de 2019, <https://eur-lex.europa.eu/legal-content/ES/TXT/?uri=celex\%3A31989L0666>.

Ministerio de Economía y Hacienda (20 de noviembre de 2007): Real Decreto 1514/2007, de 16 de noviembre, por el que se aprueba el Plan General de Contabilidad. Boletín Oficial del Estado. 278: 47402- 47407. Consultado el 3 de marzo de 2019, <https://www.boe.es/eli/es/ $\mathrm{rd} / 2007 / 11 / 16 / 1514 /$ con>.

Ministerio de Economía y Hacienda (21 de noviembre de 2007): Real Decreto 1515/2007, de 16 de noviembre, por el que se aprueba el Plan General de Contabilidad de Pequeñas y Medianas Empresas y los criterios contables específicos para microempresas. Boletín Oficial del Estado. 279:47560-47566. Consultado el 3 de marzo de 2019, <https://www.boe.es/eli/ es/rd/2007/11/16/1515>.

Ministerio de Justicia (16 de abril de 1947): Decreto de 14 de febrero de 1947 por el que se aprueba el Reglamento Hipotecario. Boletín Oficial del Estado. 106:2238-2239. Consultado el 13 de marzo de 2019, <https://www.boe.es/diario_boe/txt.php?id=BOE-A-2011-3829>.

Ministerio De Justicia (28 de febrero de 2011): Resolución de 7 de enero de 2011, de la Dirección General de los Registros y del Notariado, en el recurso interpuesto por Solesi, SRL Sucursal en España, contra la nota de calificación de la registradora mercantil y de bienes muebles X de Barcelona, por la que se deniega el depósito de las cuentas anuales de la citada compañía. Boletín Oficial del Estado. 50:23091-23094. Consultado el 13 de marzo de 2019, $<$ https://www.boe.es/diario_boe/txt.php?id=BOE-A-2011-3829>.

Parlamento Europeo y Consejo de la Unión Europea (19 de julio de 2002): Reglamento (CE) n ${ }^{\circ}$ 1606/2002 del Parlamento Europeo y del Consejo, de 19 de julio de 2002, relativo a la aplicación de normas internacionales de contabilidad. Diario Oficial de la Unión Europea. L243:0001-0004. Consultado el 13 de mayo de 2019, <https://eur-lex.europa.eu/ legal-content/ES/TXT/HTML/?uri=CELEX:32002R1606\&from=ES>.

\section{Anexo 3: Normas IFRS/NIIF y NIIF-EU}

Comisión Europea [IFRS Foundation] (3 de noviembre de 2008): Norma Internacional de Contabilidad $\mathrm{N}^{\circ}$ 1. Presentación de estados financieros. Diario Oficial de la Unión Europea. L320:5-21. Consultado el 3 de junio de 2019, <https://eur-lex.europa.eu/legal-content/ES/ ALL/?uri=CELEX:32008R1126\#d1e32-5-1>.

Comisión Europea [IFRS Foundation] (3 de noviembre de 2008): Norma Internacional de Contabilidad $\mathrm{N}^{\circ} 12$. Impuesto sobre las ganancias. Diario Oficial de la Unión Europea. L320:53-71. Consultado el 3 de junio de 2019, <https://eur-lex.europa.eu/legal-content/ES/ ALL/?uri=CELEX:32008R1126\#d1e32-72-1>.

Comisión Europea [IFRS Foundation] (3 de noviembre de 2008): Norma Internacional de Contabilidad $\mathrm{N}^{\circ} 24$. Informaciones a revelar sobre partes vinculadas. Diario Oficial de la Unión Europea. L320:146-149. Consultado el 3 de junio de 2019, <https://eur-lex.europa.eu/ legal-content/ES/ALL/?uri=CELEX:32008R1126\#d1e32-146-1>. 
Comisión Europea [IFRS Foundation] (3 de noviembre de 2008): Norma Internacional de Contabilidad $\mathrm{N}^{\circ} 36$. Deterioro del valor de los activos. Diario Oficial de la Unión Europea. L320:215-240. Consultado el 3 de junio de 2019, <https://eur-lex.europa.eu/legal-content/ ES/ALL/?uri=CELEX:32008R1126\#d1e32-215-1>.

Comisión Europea [IFRS Foundation] (3 de noviembre de 2008): Norma Internacional de Contabilidad $\mathrm{N}^{\circ}$ 38. Activos intangibles. Diario Oficial de la Unión Europea. L320:252-269. Consultado el 3 de junio de 2019, <https://eur-lex.europa.eu/legal-content/ES/ALL/?uri=C ELEX:32008R1126\#d1e33-252-1>.

IFRS Foundation (Actualizado: 29 de mayo de 2013): IAS 36 - Impairment of Assets [NIC 36 - Deterioro del valor de los activos]. IFRS. Consultado el 5 de junio de 2019, <https:// www.ifrs.org/issued-standards/list-of-standards/ias-36-impairment-of-assets/>.

IFRS Foundation (Actualizado: 12 de diciembre de 2013): IAS 24 - Related Party Disclosures [NIC 24 - Información a revelar sobre partes vinculadas]. IFRS. Consultado el 5 de junio de 2019, <https://www.ifrs.org/issued-standards/list-of-standards/ias-24-related-partydisclosures/\#about>.

IFRS Foundation (Actualizado: 12 de mayo de 2014): IAS 38 - Intangible Assets [NIC 38 Activos intangibles]. IFRS. Consultado el 5 de junio de 2019, <https://www.ifrs.org/issuedstandards/list-of-standards/ias-38-intangible-assets/>.

IFRS Foundation (Actualizado: 12 de diciembre de 2017): IAS 12 - Income Taxes [NIC 12 Impuesto sobre las ganancias]. IFRS. Consultado el 5 de junio de 2019, <https://www.ifrs. org/issued-standards/list-of-standards/ias-12-income-taxes/>.

IFRS Foundation (Actualizado: 31 de octubre de 2018): IAS 1 - Presentation of Financial Statements [NIC - Presentación de estados financieros]. IFRS. Consultado el 5 de junio de 2019, <https://www.ifrs.org/issued-standards/list-of-standards/ias-1-presentation-offinancial-statements/>.

\section{Anexo 4: Otras normas}

Financial Accounting Standards Board (Actualizado: Junio de 2019): Generally Accepted Accounting Principles (GAAP or U.S. GAAP). U.S. Securities and Exchange Commission. Consultado el 4 de abril de 2019, <https://asc.fasb.org/>.

Institute of Chartered Accountants in England and Wales (Actualizado: Mayo de 2019): FRS 102 - The Financial Reporting Standard applicable in the UK and Republic of Ireland. Consultado el 1 de marzo de 2019, <https://www.icaew.com/technical/financialreporting/uk-gaap/frs-102-the-financial-reporting-standard $>$.

Institute of Chartered Accountants in England and Wales (Actualizado: Julio de 2019): FRS 101 - Reduced Disclosure Framework. Consultado el 1 de marzo de 2019, <https:// www.icaew.com/technical/financial-reporting/uk-gaap/frs-101-reduced-disclosure-framework>.

International Auditing and Assurance Standards Board (Actualizado: 15 de enero de 2015): International Standard on Auditing (ISA) 700 (revised), Forming an Opinion and Reporting on Financial Statements. International Federation of Accountants. Consultado el 4 de abril de 2019, <https://www.iaasb.org/publications-resources/international-standardauditing-isa-700-revised-forming-opinion-and $>$. 